Bundesgesundheitsbl - Gesundheitsforsch Gesundheitsschutz 2006 • 49:1162-1168 DOI 10.1007/s00103-006-0078-5 Online publiziert: 30 . Oktober 2006

(c) Springer Medizin Verlag 2006
K. Abu-Omar · A. Rütten Institut für Sportwissenschaft und Sport, Friedrich-Alexander-Universität ErlangenNürnberg, Erlangen, BRD

\section{Sport oder körperliche Aktivität im Alltag?}

\author{
Zur Evidenzbasierung von Bewegung \\ in der Gesundheitsförderung
}

$M_{\text {it }}$ it der 2005 vom Bundesgesundheitsministerium gestarteten „3000Schritte-Kampagne“ ist das Thema „Alltagsbewegung" auch in Deutschland angekommen. International betrachtet, haben sich bereits im letzten Jahrzehnt die Bemühungen deutlich verstärkt, Bevölkerungsgruppen zu einem höheren Maß an körperlicher Aktivität im Alltag anzuregen. Man folgte damit einem Wandel in den bewegungsbezogenen Public-HealthEmpfehlungen: Während solche Empfehlungen für Erwachsene noch bis Anfang der 199oer-Jahre auf ein mindestens 3-mal pro Woche ausgeübtes spezielles gesundheitsorientiertes Fitnesstraining abzielten $[1,2]$, wird Erwachsenen seitdem zu mindestens 30 Minuten mittelschwerer körperlicher Aktivität an den meisten, wenn möglich, allen Tagen einer Woche, geraten [3]. Neuere Empfehlungen zu körperlicher Aktivität gehen sogar über 30 Minuten hinaus und betonen, dass zu einer Vermeidung von Übergewicht für Erwachsene 6o Minuten an täglicher moderater Aktivität erforderlich sind [4]. Diese neueren Empfehlungen haben die bisher geltenden nicht abgelöst, sondern sind, bezogen auf die Zielgröße Körpergewicht, als Ergänzung zu den bestehenden Empfehlungen zu verstehen [5].

Die Verschiebungen in den PublicHealth-Empfehlungen reflektieren nach Sallis, Linton, Kraft [6] auch Änderungen in der Ausrichtung der Forschung zur körperlicher Aktivität und Gesundheit insgesamt: Wurden bis zu den 1970erJahren vorwiegend die physiologischen Wirkungen einer intensiven körperlichen Aktivität studiert, schloss sich bis in die 1990er-Jahre eine Phase an, die die gesundheitlichen Wirkungen körperlicher Aktivität und deren Relevanz für Public Health erforschte. Seit den 1990er-Jahren erfolgte eine zunehmende Beschäftigung mit der Evidenzbasierung von Interventionen zur Steigerung der körperlichen Aktivität. Hier lässt sich in den letzten Jahren eine Öffnung des Forschungsfeldes hin zu anderen Wissenschaftsdisziplinen (z. B. Raumplanung) beobachten, die einen Erkenntnisgewinn über die politischen und infrastrukturellen Einflussfaktoren auf körperliche Aktivität zum Ziel haben. In diesem Kontext ist ein „aktiver Lebensstil“ (active living), gekennzeichnet durch die Integration von Bewegung in den Alltag und die bewusste Wahrnehmung von Möglichkeiten, um zu Fuß zu gehen/mit dem Rad zu fahren, in das Blickfeld des wissenschaftlichen Interesses gerückt.

Dieser Beitrag möchte nach einer begrifflichen Abgrenzung einen Überblick über die vorliegenden Evidenzen der gesundheitlichen Wirkungen der unterschiedlichen Bewegungskonzepte (Sport, Gesundheitssport, körperliche Aktivität, gesundheitsförderliche körperliche Aktivität) geben und darüber hinaus die Evidenzen unterschiedlicher Ansätze zur Förderung von Sport und körperlicher Aktivität vorstellen. Bezogen auf die deutsche Situation, möchte er hierdurch aktuelle Entwicklungen zur Etablierung von Gesundheitssportprogrammen und Thematisierung von gesundheitsförderlichen körperlichen Alltagsaktivitäten unterstützen [7].

\section{Begriffliche Abgrenzungen}

Der Begriff „körperliche Aktivität“ (physical activity) bezieht sich auf körperliche Bewegung, die durch die Skelettmuskulatur produziert wird und den Energieverbrauch über den Grundumsatz anhebt [8]. Körperliche Aktivität wird gegenüber Sport, Gesundheitssport und gesundheitsförderlicher körperlicher Aktivität zumeist als Oberbegriff verwendet. Im europäischen Raum hat sich Ende der 1990er-Jahre der Begriff der gesundheitsförderlichen körperlichen Aktivität (Health-Enhancing Physical Activity: HEPA) etabliert. Gesundheitsförderliche körperliche Aktivität bezeichnet hierbei jede Form körperlicher Aktivität, die einen gesundheitlichen Nutzen verspricht und kein übermäßiges gesundheitliches Risiko beinhaltet [9]. Im US-amerikanischen Raum wird hierfür in neuerer 
Zeit auch der Begriff aktiver Lebensstil (active living) verwendet, um eine Erweiterung von Freizeitaktivitäten hin zu in den Alltag integrierten Aktivitäten (z. B. zu Fuß gehen, Radfahren zum Transport) zu beschreiben [10].

Demgegenüber bezeichnet "Sport“ eine historisch-kulturell definierte Untergruppe von „körperlicher Aktivität“, für die traditionell insbesondere körperliche Leistung, Wettkampf und Spaß an der Bewegung typisch sind. Im Angelsächsischen wird der Begriff der „leisure-time physical activity" verwendet, um die Untergruppe der körperlichen Aktivitäten in der Freizeit zu beschreiben, oder aber der Begriff „exercise“, um auf ein strukturiertes körperliches Trainingsprogramm hinzuweisen.

In der deutschen sportwissenschaftlichen Diskussion wurde in den letzten Jahren eine eindeutige Abgrenzung zwischen "Sport" und „Gesundheitssport" vorgenommen. Gesundheitssport wird dabei als ein hoch strukturierter, auf gesundheitliche Effekte ausgerichteter Ausschnitt aus gesundheitsförderlicher körperlicher Aktivität im Schnittbereich von Sport- und Gesundheitssystem verstanden [11]. Als spezifische Ziele und Qualitäten des Gesundheitssports werden die Stärkung physischer und psychosozialer Ressourcen, die Prävention von Risikofaktoren, die Bewältigung von Gesundheitsbeschwerden, die Bindung an gesundheitssportliches Verhalten sowie die Verbesserung der Bewegungsverhältnisse definiert. Die vorgenommenen begrifflichen Abgrenzungen ermöglichen eine Betrachtung der Evidenzbasierung der gesundheitlichen Wirkungen verschiedener Bewegungskonzepte.

\section{Konzepte der Evidenzbasierung}

In der Medizin wird Evidenzbasierung als „[...] the conscientious, explicit, and judcious use of current best evidence in making decisions about the care of individual patients" [12] definiert. Geeignete methodische Verfahren für einen Erkenntnisgewinn sind aus dieser Sichtweise heraus randomisierte und kontrollierte Studien. Dabei wird die Qualität von Studien nach festgelegten Gütekriterien beurteilt, als deren höchste Stufe Metaanalysen über

Bundesgesundheitsbl - Gesundheitsforsch - Gesundheitsschutz 2006 • 49:1162-1168 DOI 10.1007/s00103-006-0078-5

(c) Springer Medizin Verlag 2006

\section{K. Abu-Omar · A. Rütten}

\section{Sport oder körperliche Aktivität im Alltag? Zur Evidenzbasierung von Bewegung in der Gesundheitsförderung}

\section{Zusammenfassung}

In den letzten Jahren haben sich international die Public-Health-Bemühungen um eine körperliche Aktivierung der Bevölkerungen verstärkt. Hierbei wird sowohl auf Konzepte einer sportlichen Aktivierung als auch einer körperlichen Aktivierung im Alltag zurückgegriffen. Der Beitrag vergleicht die vorliegenden Evidenzen der gesundheitlichen Wirkungen der Konzepte körperlicher Aktivität, gesundheitsförderlicher körperlicher Aktivität, Sport und Gesundheitssport. Anhand vorliegender Reviews und Meta-Analysen werden darüber hinaus die Evidenzen der verschie- denen Strategien einer individuums- bzw. populationsbezogenen körperlichen Aktivierung vorgestellt und diskutiert. Bezogen auf die deutsche Situation, möchte dieser Beitrag aktuelle Entwicklungen zur Etablierung von Gesundheitssportprogrammen und der Thematisierung gesundheitsförderlicher körperlicher Alltagsaktivitäten unterstützen.

\section{Schlüsselwörter}

Körperliche Aktivität · Public Health . Gesundheit · Sport · Evidenzen

\section{Physical activity and health. Evidence for the health benefits of different physical activity promotion concepts}

\begin{abstract}
Recent years have been marked by increased efforts of public health to promote physical activity. Concepts used for the promotion of physical activity range from the prescription of exercise to advice for "active living". The article compares existing evidence for the health benefits of the concepts of physical activity, healthenhancing physical activity, exercise and the German concept of "health sport". Furthermore, existing evidence for different
\end{abstract}

promotion strategies for physical activity on the individual and population level are presented and discussed. Referring to the situation in Germany, the article intends to support current discussions regarding the development of health sport programs and initiatives to promote active living.

\section{Keywords}

physical activity - public health $\cdot$ health . exercise - evidence-base 
verschiedene randomisierte und kontrollierte Studien gelten [13].

Im Bereich der Gesundheitsförderung ist die Anwendung einer medizinischen Methodik des Erkenntnisgewinns umstritten. Zentrale Kritikpunkte, die gegen eine Anwendung von randomisierten Studiendesigns in der Gesundheitsförderung sprechen, sind demnach deren geringe Eignung für die Evaluation gemeindeund populationsbezogener Interventionen sowie von Interventionen, die auf Politikveränderungen abzielen [14]. Darüber hinaus wird deren Unzulänglichkeit moniert, Einblicke in die Erfahrung der Betroffenen zu geben [15] und Aussagen über die Übertragbarkeit von Studienergebnissen auf andere Zielgruppen oder Settings zu treffen [16]. Auf internationaler Ebene sind daher Aktivitäten unternommen worden, um für den Bereich der Gesundheitsförderung angemessenere Methoden des (evidenzbasierten) Erkenntnisgewinns zu entwickeln. Diese Verfahren nutzen sowohl quantitative als auch qualitative Methoden und sind auch für die Evaluierung gemeindebezogener, settingbezogener und politikfeldbezogener Maßnahmen der Gesundheitsförderung anwendbar [17].

Für den Bereich der Sportwissenschaft lässt sich sowohl die Verwendung medizinisch orientierter als auch an New Public Health orientierter Evaluationsmethodiken verzeichnen. Studien zur Erforschung physiologischer Wirkungen verschiedener Bewegungskonzepte richten sich in der Sportwissenschaft vorwiegend an einer Methodik der evidenzbasierten Medizin aus. Auch zur Evaluation individuumsbezogener Maßnahmen der körperlichen Aktivierung werden diese Verfahren häufig eingesetzt. Auf der anderen Seite finden, z. B. für den Bereich der populations- und gemeindebezogenen Studien zur körperlichen Aktivierung, auch andere in der Sportwissenschaft entwickelte und an der Gesundheitsförderung orientierte Evaluationsmethodiken Anwendung [18, 19, 20].

\section{Evidenzbasierte gesundheitliche Wirkungen der verschiedenen Bewegungskonzepte}

Bei dem Versuch einer differenzierten Betrachtung der evidenzbasierten Wirkungen der verschiedenen Bewegungskonzepte auf die Gesundheit entstehen verschiedene Probleme. Diese beziehen sich zum einen auf die oftmals nicht vorgenommene Abgrenzung der Bewegungskonzepte (situativer Rahmen und Art der Aktivität) innerhalb von Studien, zum anderen auf die Notwendigkeit, Parameter des Volumens der Aktivität (Häufigkeit, Dauer und Intensität) in eine Betrachtung mit einbeziehen zu müssen.

Für körperliche Aktivität, im Sinne eines konzeptuellen Oberbegriffs zur Beschreibung der Summe aller Aktivitäten, sind in den letzten Jahrzehnten starke Evidenzen eines gesundheitlichen Nutzens im Hinblick auf verschiedene Erkrankungsbilder erarbeitet worden [8, 21, 22]. Nach diesen Reviews kann es als nachgewiesen gelten, dass körperliche Aktivität (gegenüber körperlicher Inaktivität) das Risiko für kardiovaskuläre Erkrankungen, Darmkrebs und Diabetes mellitus Typ II senken kann und in der Lage ist, die Lebenserwartung zu steigern. Darüber hinaus verbessert körperliche Aktivität die Knochendichte und den Zustand des Muskel-Skelett-Systems und kann so die Kompetenzen der Alltagsbewältigung im Alter steigern. Ebenfalls nachgewiesen sind die positiven Wirkungen körperlicher Aktivität auf das Wohlbefinden und die Lebensqualität. Betrachtet man das notwendige Volumen körperlicher Aktivität für einen gesundheitlichen Nutzen, so sind die Evidenzen für die positiven gesundheitlichen Effekte moderater Intensitäten durch neuere Studien weiter gestärkt worden [23].

Weniger eindeutige Zusammenhänge lassen sich zwischen körperlicher Aktivität und der psychischen Gesundheit (Angstzustände, Depressionen) und körperlicher Aktivität und dem Körpergewicht nachweisen. Zwar wird vielfach eine positive Wirkung körperlicher Aktivität auf Angstzustände und Depressionen angenommen, jedoch liegen für diese angenommenen Zusammenhänge widersprüchliche Studienergebnisse vor, sodass Reviews und Metaanalysen keinen eindeutigen Nachweis erbringen $[24,25]$. Auch die Reduktion des Körpergewichts durch körperliche Aktivität wird vielfach aufgrund der Ergebnisse cross-sektionaler Studien angenommen. Dem steht gegenüber, dass Interventionsstudien in der Regel nur relativ geringe Effekte gesteigerter körperlicher Aktivität (zumeist durch ein strukturiertes Trainingsprogramm) auf eine Reduktion des Körpergewichts nachweisen [26]. Auf der anderen Seite verdichten sich die Hinweise, dass nicht ein Rückgang des Sporttreibens, sondern der Alltagsaktivitäten für die beobachtbare Zunahme an Übergewicht und Adipositas in der Bevölkerung verantwortlich sind [27]. Hieraus lässt sich schließen, dass gesteigertes Sporttreiben einer Gewichtszunahme vorbeugen kann, aber ohne komplementäre Maßnahmen im Bereich der Alltagsbewegung für eine dauerhafte bevölkerungsweite Gewichtsreduktion nicht ausreichend ist [22].

Eine weitere Differenzierung der gesundheitlichen Wirkungen körperlicher Aktivität hinsichtlich des situativen Rahmens und der Art der Aktivität liefert keine eindeutigen Befunde. Medizinischphysiologisch orientierte Wirkungsmodelle führen die beobachtbaren positiven gesundheitlichen Einflüsse körperlicher Aktivität auf den durch diese Aktivität verursachten erhöhten Kalorienverbrauch und eine Verringerung des Blutdrucks zurück [8]. Folgt man dieser Argumentation, so sollte der situative Rahmen körperlicher Aktivität keinen großen Einfluss auf deren gesundheitliche Wirkungen haben. Verschiedene Studien legen demgegenüber aber die Bedeutung des situativen Rahmens für deren gesundheitliche Wirkungen nahe: Körperliche Aktivitäten in der Freizeit bzw. zum Transport (zu Fuß gehen, Rad fahren) senken das Schlaganfallrisiko deutlicher als während der Arbeit [28]. Ebenso sind für Frauen positive gesundheitliche Effekte durch Freizeitaktivitäten, nicht aber durch Hausarbeiten nachgewiesen [29]. Allerdings stehen bisher noch zu wenige Studien zur Verfügung, die konkrete Aussagen über den Einfluss des situativen Rahmens auf die gesundheitlichen Effekte körperlicher Aktivität zulassen. 
Betrachtet man Evidenzen zu den gesundheitlichen Wirkungen des Unterbereichs der gesundheitsförderlichen körperlichen Aktivität, so sind hier in den letzten Jahren verstärkt positive Nachweise erbracht worden. Dies bezieht sich vor allem auf die gesundheitsförderlichen Wirkungen von Aktivität zum Transport (zu Fuß gehen, Rad fahren). So ist häufiges zu Fuß gehen mit einem niedrigeren Körperfettanteil [30], einem niedrigeren Blutdruck [31] und auch einem geringeren Risiko für kardiovaskuläre Erkrankungen [23] in Verbindung gebracht worden. Reviews zu diesem Thema sehen große Potenziale der körperlichen Aktivität zum Transport für die populationsbezogene Gesundheitsförderung [32, 33].

Schränkt man die Betrachtung auf den Bereich des Sports ein, so lassen sich hier keine generalisierbaren Aussagen über positive Wirkungen treffen. Dies hängt damit zusammen, das nur über eine Differenzierung der Belastungen auf der einen und der gesundheitlichen Wirkungen auf der anderen Seite eine Beschreibung der Effekte des Sports möglich ist. So haben Metaanalysen keine nachweisbaren $\mathrm{Zu}$ sammenhänge zwischen dem generellen Sporttreiben und der physischen [34] und psychischen Gesundheit [35] feststellen können.

Der gesundheitliche Nutzen von spezifischen Gesundheitssportprogrammen ist demgegenüber eindeutig dokumentiert. So ließen sich bei den Teilnehmern an Gesundheitssportprogrammen u. a. nachhaltige Effekte, bezogen auf die körperliche Fitness und die psychische Gesundheit, feststellen $[36,37]$. Diese sind darauf zurückzuführen, dass sportliche Aktivität im Rahmen eines Gesundheitssportprogramms qualitätsgesichert und an gesundheitlichen Kernzielen orientiert durchgeführt wird.

Zusammenfassend kann festgehalten werden, dass ein Gesamtnutzen körperlicher Aktivität dokumentiert ist, darüber hinaus aber eine differenziertere Betrachtung hinsichtlich spezifischer gesundheitlicher Effekte notwendig erscheint. Für gesundheitsförderliche Aktivitäten und den Bereich des Gesundheitssports bestehen nachgewiesene positive Zusammenhänge zu Gesundheitsparametern, während für körperliche Aktivitäten in den Kontexten
Berufs- oder Hausarbeit sowie des Sports allgemein positive gesundheitliche Wirkungen nicht ohne weiteres angenommen werden können. Eine weitere Differenzierung der Kontexte und des Volumens körperlicher Aktivität für die Beschreibung der gesundheitlichen Wirkungen erscheint daher nötig.

\section{Evidenzbasierte Interventionen zur Förderung von körperlicher Aktivität}

Ziel einer Analyse der evidenzbasierten Wirkungen von Interventionen sollten Aussagen darüber sein, welche Maßnahmen, in welchen Kontexten, für welche Zielgruppen geeignet erscheinen, spezifische Effekte hervorzurufen [38]. Für Interventionen mit dem Ziel einer körperlichen Aktivierung sind als beschreibende Dimensionen zu den Maßnahmen ferner beispielsweise von Interesse, welche Bewegungskonzepte sie verwenden (z. B. Aktivierung im Alltag gegenüber regelmäßigem Ausdauersport), über welches Medium sie transportiert werden (z. B. Internet gegenüber persönlichen Kontakten), welche Dauer und welche Kontaktintensität sie haben (z. B. mehrmals wöchentlich gegenüber alle 3 Monate), und auf welcher Ebene Effekte angestrebt werden (z. B. Wissensänderungen gegenüber Verhaltensänderungen).

Zwar existiert eine Reihe von Sekundäranalysen über Interventionen zur Förderung von Sport und körperlicher Aktivität, jedoch können anhand dieser nur sehr begrenzt Aussagen zu den oben genannten Fragestellungen abgeleitet werden. Dies hängt mit Problemen in der konzeptionellen Abgrenzung von Interventionsinhalten sowie mit der für die Durchführung von Sekundäranalysen verwendeten Methodik zusammen. So unterscheiden die existierenden Sekundäranalysen zumeist nicht zwischen Ansätzen zur Förderung von Bewegung im Alltag und Ansätzen zur Förderung des Sporttreibens; auch weil vorliegende Studien zum Teil beide Ansätze komplementär verwenden. Sekundäranalysen selegieren Studien in der Regel aufgrund eines als methodisch hochwertig erachteten Studiendesigns (randomisierte kontrollierte Studien), was dann oftmals zum
Ausschluss eines Großteils der existierenden Untersuchungen führt - und zwar ohne weitere Begutachtung der Qualität der vorgenommenen Interventionsmaßnahmen.

Für die folgende Darstellung zur Evidenzbasierung verschiedener Interventionen im Bereich Bewegung und Gesundheit wird zwischen individuumsbezogenen Ansätzen (mit Maßnahmen, die sich auf ausgewählte Individuen beziehen) und populationsbezogenen Ansätzen (mit Maßnahmen, die sich an ganze Bevölkerungen bzw. Teilbevölkerungen) richten, unterschieden [39].

\section{Individuumsbezogene Ansätze zur Gesundheitsförderung durch körperliche Aktivität}

In einer Sekundäranalyse von Studien zur körperlichen Aktivität haben die Centers for Disease Control and Prevention (CDC) $[40,41]$ auch eine Reihe individuumsbezogener Interventionen untersucht. Nach den CDC bestehen demnach keine hinreichenden Beweise für die Wirksamkeit gesundheitserzieherischer Maßnahmen zur Steigerung körperlicher Aktivität im Kontext von Schule oder College. Individuell adaptierte Verhaltensänderungsprogramme, wie sie z. B. im Rahmen eines Gesundheitssportangebotes durchgeführt werden, gelten nach der Analyse des CDC dagegen als evidenzbasiert und werden als Strategie für eine körperliche Aktivierung empfohlen.

Weitere Sekundäranalysen beziehen sich auf die Wirksamkeit individuumsbezogener Interventionsansätze im Kontext des Gesundheitssystems und der Arbeitswelt. Eden et al. [42] konnten in ihrer Metaanalyse keinen eindeutigen Nachweis erbringen, dass eine ärztliche Beratung zum Thema körperliche Aktivität und Sport Auswirkungen auf das Verhalten der Patienten hat. Zumindest kurzfristige Effekte für Intervention in diesem Kontext sehen hingegen Hillsdon et al. [43]. Im Hinblick auf die Arbeitswelt sind aufgrund von methodischen Mängeln nur wenige Studien in Sekundäranalysen mit einbezogen worden, die übereinstimmend keine eindeutige Wirkung von Interventionen zur Steigerung körperlicher Aktivität nachweisen [44, 45]. 
Über die zielgruppenspezifische Wirkung individuumsbezogener Interventionen liegen verschiedene Sekundäranalysen vor. Für die Gruppe der Senioren kommen entsprechende Untersuchungen $\mathrm{zu}$ dem Schluss, dass es zum einen auch für diese gelingen kann, die körperliche Aktivität zu steigern [46] und zum anderen Heimtrainingsprogramme für die Erzielung nachhaltigerer Effekte eher geeignet erscheinen als im Verein oder Fitnesscenter verortete Programme [47]. Bei der Zielgruppe der Kinder und Jugendlichen bestehen Hinweise auf eine Wirksamkeit von Interventionen zur Steigerung der körperlichen Aktivität. Allerdings werden auch hier aufgrund methodischer Kriterien nur wenige Studien in die Sekundäranalysen mit einbezogen [48]. Betrachtet man sozial benachteiligte Zielgruppen (z. B. untere Schichten, ethnische Minoritäten), so lässt sich hier aufgrund der vorliegenden Sekundäranalysen keine Wirksamkeit individuumsbezogener Interventionen nachweisen [49]. Eine Gesamtbeurteilung der Effekte individuumsbezogener Interventionen zur Steigerung körperlicher Aktivität hat die Cochrane Library verfasst. Nach diesem Review haben die berücksichtigten Studien insgesamt positive Effekte auf die körperliche Fitness und das subjektive $\mathrm{Ma} ß$ an körperlicher Aktivität ergeben $[43,50]$.

Aufgrund der oben angesprochenen konzeptionellen und methodischen Begrenzungen der vorliegenden Studien und Sekundäranalysen ist es derzeit aus wissenschaftlicher Sicht schwierig, eindeutige Empfehlungen für oder gegen bestimmte Interventionen anzugeben. Vieles deutet jedoch darauf hin, dass bestimmte individuumsbezogene Maßnahmen eine größere Wirkungswahrscheinlichkeit aufweisen. Insbesondere für individuellverhaltensorientierte Interventionen, die eine Steigerung körperlicher Aktivität und spezifische gesundheitliche Effekte durch die Teilnahme an einem strukturierten Gesundheitssportprogramm anstreben, besteht eine nachgewiesene Wirksamkeit. Teilnehmer an dieser Art von Programmen konnten z. B. ihren Kalorienverbrauch um ca. $64 \%$ und ihre für körperliche Aktivitäten aufgewendete Zeit um ca. $35 \%$ steigern [41].
Bezogen auf Deutschland sind die Voraussetzungen für den weiteren Ausbau qualitätsgesicherter Gesundheitssportprogramme in den letzten Jahren weiter verbessert worden. Deutschland verfügt insbesondere durch das organisierte Vereinswesen und die bestehenden Qualitätssiegel für den Gesundheitssport der großen Sportverbände (z. B. DSB, DTB) über sehr gute Bedingungen für einen weiteren Ausbau individueller gesundheitssportlicher Programme.

\section{Populationsbezogene Ansätze zur Gesundheitsförderung durch körperlicher Aktivität}

Bei den populationsbezogenen Interventionen unterscheiden die CDC in der bereits angeführten Sekundäranalyse zwischen Interventionen mit Hilfe von Informationsangeboten und umwelt- und politikbezogenen Ansätzen [40, 41].

Für Informationsangebote ergibt die Analyse ein differenziertes Bild: Demnach bestehen für Interventionen, die durch gezielt in die alltägliche Lebens- und Bewegungswelt eingebundene Informationen - z. B. Schild: „Benutze Treppe statt Aufzug" - eine Steigerung der körperlichen Aktivität erreichen wollen, hinreichende Evidenzen. Demgegenüber wurde für Massenmedienkampagnen bisher keine hinreichende Wirksamkeit für eine nachhaltige Verhaltensänderung im Bezug auf körperliche Aktivität nachgewiesen [40, 41]. Dass sich durch massenmediale Kampagnen zwar Wissensveränderungen, aber keine Verhaltensänderungen erzielen lassen, wird auch durch andere Sekundäranalysen bestätigt $[51,52]$.

Durchgehend positiv werden nach den vorliegenden wissenschaftlichen Erkenntnissen umwelt- und politikbezogene Ansätze zur Förderung körperlicher Aktivität beurteilt. Diese Ansätze versuchen eine Steigerung körperlicher Aktivität durch die Verbesserung der Infrastrukturen für diese (z. B. Bau von Radwegen) oder durch diese unterstützende politische Maßnahmen (z. B. steuerliche Anreize für Radverkehr) [33] zu erreichen. Sowohl die CDC [40, 41], als auch Cavill und Foster [53] sehen in solchen Ansätzen große Potenziale für die populationsbezogene Förderung körperlicher Aktivität.
Während jedoch die Umsetzung umwelt- und politikbezogener Interventionsstrategien - nicht zuletzt wegen Schwierigkeiten in der Zusammenarbeit unterschiedlicher Politikfelder - nur schleppend vorankommt, finden in vielen Ländern, so auch in Deutschland, primär massenmedial fokussierte Kampagnen zur Förderung der körperlichen Aktivität immer noch Verbreitung. Wie einleitend erwähnt, ist im Jahr 2005 vom Bundesministerium für Gesundheit eine Kampagne zur Förderung von Alltagsaktivitäten gestartet worden (300o Schritte extra - einfach gesünder). Diese empfiehlt Erwachsenen das Gehen von 3000 zusätzlichen Schritten täglich (überwacht durch einen Schrittzähler) und weicht in der inhaltlichen Konzeption von den international üblichen „10.00o Schritte am Tag"-Kampagnen ab. Insbesondere das fehlende Setzen einer Bewegungsbenchmark (10.00o Schritte), die den Teilnehmern eine Rückmeldung über das eigene Bewegungsverhalten im Vergleich zu den Empfehlungen geben könnte, ist in dieser Kampagne zu hinterfragen. Mit dieser Kampagne wird zwar ein gesundheitspolitisch relevantes neues Thema aufgegriffen (wissenschaftlicher Nachweis der gesundheitlichen Effekte von Alltagsbewegung liegt vor, s. oben; speziell zum international üblichen „10.000-SchritteAnsatz" [31]), dieses allerdings in Form einer primär massenmedial ausgerichteten Kampagne - z.B. über die Verteilung von Schrittzählern und Informationsmaterial - der Bevölkerung nahe gebracht (kein wissenschaftlicher Nachweis für die verhaltensrelevante Wirksamkeit dieser Interventionsform, s. oben).

\section{Diskussion}

Dieser Beitrag hat vorliegende Evidenzen zur Gesundheitsförderung durch körperliche Aktivität betrachtet, wobei eine Unterscheidung hinsichtlich der unterschiedlichen Bewegungskonzepte (körperliche Aktivität, gesundheitsförderliche körperliche Aktivität, Sport, Gesundheitssport) vorgenommen wurde. Im Bezug auf die gesundheitlichen Wirkungen konnte festgestellt werden, dass für die Gesamtheit körperlicher Aktivitäten zwar ein gesundheitlicher Nutzen deutlich nachgewiesen 
ist, jedoch daraus nicht automatisch auf einen gesundheitlichen Nutzen jeder Art körperlicher Aktivität geschlossen werden kann. Insbesondere für berufliche körperliche Aktivitäten oder das klassische Sporttreiben sollte ein solcher Nutzen nicht von vornherein unterstellt werden. Demgegenüber kann ein gesundheitlicher Nutzen von strukturierten Gesundheitssportprogrammen und auch Alltagsaktivitäten in der Freizeit oder zum Transport (z. B. zu Fuß gehen, Rad fahren) aufgrund der Befundlage angenommen werden. Den Alltagsaktivitäten wird in diesem Zusammenhang gerade für eine Prävention des Übergewichts zukünftig ein hoher Stellenwert eingeräumt.

Geeignete Strategien zur Förderung körperlicher Aktivität sind zum einen individuell-verhaltensorientierte strukturierte Trainingsprogramme, für die Evidenzen für eine erfolgreiche körperliche Aktivierung bestehen. Dem in Deutschland verbreiteten qualitätsgesicherten Gesundheitssport kann in diesem Zusammenhang aufgrund der vorliegenden Studien eine Wirksamkeit unterstellt werden. Auf der anderen Seite bestehen bei dieser Art individuumsbezogener Programme durch die oftmalige Selbstselektion der Teilnehmer Probleme hinsichtlich des Zugangs unterschiedlicher Subpopulationen (z. B. untere soziale Schichten, ethnische Minoritäten). Gerade durch populationsbezogene Ansätze, wie z. B. umwelt- und politikbezogene Maßnahmen, bestehen demgegenüber Potenziale, neben breiteren Bevölkerungsschichten auch Subpopulationen zu erreichen. Für umwelt- und politikbezogene Maßnahmen haben sich die Evidenzen in den letzten Jahren deutlich herauskristallisiert, auch wenn für diese nach wie vor geeignete Evaluationskonzepte zu entwickeln sind.

Für die Zukunft der Gesundheitsförderung durch körperliche Aktivierung in Deutschland ergeben sich aus den vorliegenden Befunden einige Hinweise. Ein weiterer Ausbau der vorhandenen Strukturen zur Durchführung qualitätsgesicherter Gesundheitssportprogramme erscheint empfehlenswert. In diesem Bereich kann Deutschland auch international eine führende Position einnehmen. In Bezug auf die individuumsbezogenen Maßnahmen zur körperlichen Aktivie- rung sollten zusätzlich Anstrengungen unternommen werden, durch gezielte und effiziente neue Technologien (z. B. über Telefon, Internet) weitere Zielgruppen $\mathrm{zu}$ erreichen. International sind die Potenziale dieser Interventionen zum Erreichen auch größerer Populationen betont worden [54]. Auch konnte durch Einzelstudien belegt werden, dass bereits Maßnahmen zur körperlichen Aktivierung existieren, die mit einer geringen Kontaktintensität und somit einer hohen Kosteneffizienz eine Steigerung der körperlichen Aktivität erreichen konnten [43].

Im internationalen Vergleich sind die Ausgangsbedingungen für eine populationsbezogene Bewegungsförderung durch den Aufbau geeigneter Infrastrukturen gerade für den Bereich der Alltagsaktivitäten (zu Fuß gehen, Rad fahren) in Deutschland ebenfalls sehr günstig [55]. Für nationale Initiativen, die eine weitere Förderung des Radverkehrs und des Zufußgehens aus einer gesundheitsorientierten Perspektive heraus begreifen, fehlt allerdings in Deutschland zurzeit noch die strukturelle Basis. Vor allem die für solche Initiativen erforderlichen intersektoralen Bündnisse aus Gesundheits- und Verkehrspolitik sollten unseres Erachtens auch hier in den nächsten Jahren deutlich ausgebaut werden.

Zur populationsbezogenen Bewegungsförderung ist in den letzten Jahren verstärkt die Implementierung intersektoraler und feldübergreifender Politiken angeregt worden. Diese überspannen verschiedene Bewegungskonzepte und betreiben sowohl eine Förderung der gesundheitssportlichen Aktivitäten als auch Alltagsaktivitäten. Es werden im Idealfall Maßnahmen auf verschiedenen Einflussebenen miteinander verknüpft und auf diese Weise eine körperliche Aktivierung der Menschen in verschiedenen Lebensbereichen angestrebt [10]. Eine Reihe von Ländern verfügt mittlerweile über nationale Strategien zur Förderung körperlicher Aktivität. Hervorzuheben sind hier z. B. Australien oder die Schweiz [56] sowie die Niederlande und Finnland [57].

In Deutschland scheinen bisher die föderale Länderhoheit über den Bereich des Breitensports sowie nicht geklärte Zuständigkeiten für das Thema Alltagsbewegung einer nationalen Strategie zur körperlichen
Aktivierung der Bevölkerung entgegenzustehen. Verstärkte Anstrengungen zur intersektoralen politischen Zusammenarbeit zum Thema körperlicher Aktivität wären schon aufgrund der Erfolge, die in anderen Ländern bereits erzielt wurden, wünschenswert.

Darüber hinaus ist ein verstärktes Bemühen um die Durchführung evidenzbasierter Kampagnen zur körperlichen Aktivierung in Deutschland dringend erforderlich. Derzeit durchgeführte Kampagnen legen oftmals ein relativ hohes Gewicht auf eine gelungene mediale Darstellung. Demgegenüber wird einer wissenschaftlich begründeten inhaltlichen Konzeption und vor allem dem Bemühen um einen wissenschaftlich abgesicherten Nachweis der Wirkungen meist wenig Priorität eingeräumt.

\section{Korrespondierender Autor}

\section{Karim Abu-Omar, Ph.D.}

Institut für Sportwissenschaft und Sport Friedrich-Alexander-Universität

Erlangen-Nürnberg

Gebbertstraße 123b

91058 Erlangen, BRD

E-Mail: karim@sport.uni-erlangen.de

\section{Literatur}

1. American College of Sports Medicine (1978) The recommended quantity and quality of exercise for developing and maintaining fitness in healthy adults. Med Sci Sports 10:VII-X

2. American College of Sports Medicine (1990) Position stand: the recommended quantity and quality of exercise for developing and maintaining cardioresiratory and muscular fitness in healthy adults. Med Sc Sports Exercise 22:265-274

3. Pate RR, Pratt M, Blair SN et al. (1995) Physical activity and public health: a recommendation from the Centers for Disease Control and prevention and the American College of Sports Medicine. J Am Medical Association 273:402-407

4. Institute of Medicine of the National Academies of Science (2002) Dietary reference intakes for energy, carbohydrate, fiber, fat, fatty acids, cholesterol, protein, and amino acids (macronutritients). National Academy Press, Washington DC

5. Blair SN, LaMonte MJ, Nichaman MZ (2004) The evolution of physical activity recommendations: how much is enough? Am J Clin Nutrition 79: 913S-920S

6. Sallis JF, Linton L, Kraft MK (2005) The first active living research conference. Growth of a transdisciplinary field. Am J Prev Med 28(2S2):93-95

7. Rütten A, Abu-Omar K, Lampert T, Ziese T (2005) Gesundheitsberichterstattung des Bundes. Körperliche Aktivität, Heft 26. Robert Koch-Institut, Berlin 
8. US Department of Health and Human Services (1996) Physical activity and health: a report of the Surgeon General. Centers for Disease Control and Prevention, Atlanta

9. Foster C (2000) Guidelines for health-enhancing physical activity promotion programs. British heart foundation health promotion research group. University of Oxford Press, Oxford

10. Sallis JF, Cervero R, Ascher WW et al. (2006) An ecological approach to creating active living communities. Ann Rev Public Health 27:297-322

11. Brehm W, Bös K, Opper E (2002) Gesundheitssportprogramme in Deutschland. Hofmann, Schorndorf

12. Sackett DL, Rosenberg WMC, Gray MJ et al. (1996) Evidence based medicine: what it is and what it isn't. Br Medical J 312:71-72

13. NHS Centre for Reviews and Dissemination (1996) Review of the research on the effectiveness of health service interventions to reduce variations in health: Part 1. York: NHS Center for Reviews and Dissemination

14. Speller V, Learmouth A, Harrison D (1997) The search for evidence of effective health promotion. Br Medical J 315:361-363

15. Raphael D (2000) The question of evidence in health promotion. Health Promotion International 15:355-367

16. Pawson R, Tilley N (1997) Realistic evaluation. Sage, London

17. Rootman, I, Goodstadt M, Hyndman B et al. (2001) Evaluation in healh promotion. Principles and perspectives. WHO Regional Publication, European Series Nr. 92. WHO, Denmark

18. Rütten A (2001) Evaluating healthy public policies in community and regional contexts. In: Rootman I, Goodstadt M, Hyndman B et al. (eds) Evaluation in healh promotion. Principles and perspectives. WHO Regional Publication, European Series Nr. 92. WHO, Denmark, pp 341-364

19. US Department of Health and Human Services (2002) Physical activity evaluation handbook. US Department of Health and Human Services, Centers for Disease Control and Prevention, Atlanta

20. Martin SL, Heath GW (2006) A six-step model for evaluation of community-based physical activity programs. Preventing Chronic disease [Online], Januar; http://www.cdc.gov/pcd/issues/2006/ jan/05_0111.htm

21. Sallis JF, Owen N (1998) Physical activity and behavioral medicine. Sage, Thousand Oaks

22. Vuori I (2004) Physical inactivity as a disease risk and health benefits of increased physical activity. In: Oja P, Borms J (eds) The multidisciplinary series of physical education and sport science. Vol. 6: Perspectives health enhancing physical activity. Meyer \& Meyer, Oxford

23. Bauman AE (2004) Updating the evidence that physical activity is good for health: an epidemiological review 2000-2003. J Sc Med Sport 7(S):6-19

24. Paluska SA, Schwenk TL (2000) Physical activity and mental health. Sports Med 29:167-180

25. Lawlor DA, Hopker W (2001) The effectiveness of exercise as an intervention in the management of depression: systematic review and meta-regression analysis of randomised controlled trials. $\mathrm{Br}$ Medical J 31:763-767

26. Bensinhom DR, Kraus WE, Donahue MP, Durham MD (2006) Obesity and physical activity: a review. Am Heart J 151:598-603

27. Brownson RC, Boehmer TK, Luke DA (2005) Declining rates of physical activity in the United States: What are the contributors? Ann Rev Public Health 26:421-443
28. Hu G, Sarti C, Jousilahti P et al. (2005) Leisure time, occupational, and commuting physical activity and the risk of stroke. Stroke 36:1994-1999

29. Lawlor DA, Taylor M, Bedford C, Ebrahim S (2002) Is housework good for health? Levels of physical activity and factors associated with activity in elderly women. Results from the British Women's Heart and Health Study. J Epidemiol Community Health 56:473-478

30. Thompson DL, Rakow J, Perdue SM (2004) Relationship between accumulated walking and body composition in middle-aged women. Med Sci Sports Exercise 36:911-914

31. Iwane M, Arita M, Tomimoto S et al. (2000) Walking 10,000 steps/day or more reduces blood pressare and sympathetic nerve activity in mild essential hypertension. Hypertension Res 23:573-580

32. Dekoster J, Schollaert U (1999) Cycling: the way ahead for towns and cities. Office for official publications of the European Commission, Luxemburg

33. World Health Organisation (2002) A physically active life through everyday transport with a special focus on children and older people and examples and approaches from Europe. WHO Regional Office for Europe, Kopenhagen

34. Knoll M (1997) Sporttreiben und Gesundheit. Eine kritische Analyse vorliegender Befunde. Hofmann, Schorndorf

35. Schlicht W (1994) Sport und Primärprävention. Hogrefe, Göttingen

36. Brehm W, Janke A, Sygusch R, Wagner P (2006) Gesund durch Gesundheitssport. Zielgruppenorientierte Konzeption, Durchführung und Evaluation von Gesundheitssportprogrammen. Juventa, Weinheim

37. Brehm W, Wagner P, Sygusch R et al. (2005) Health promotion by means of health sport - a framework and a controlled intervention study with sedentary adults. Scand J Med Sci Sport 15:13-20

38. Fuchs R (2003) Sport, Gesundheit und Public Health. Hogrefe, Göttingen

39. Rütten A, Abu-Omar K (2004) Prävention durch Bewegung. Zur Evidenzbasierung von Interventionen zur Förderung körperlicher Aktivität. Z Gesundheitswissenschaften 11:229-246

40. Kahn EB, Ramsey LT, Brownson RC et al. (2002) The effectiveness of interventions to increase physical activity. A systematic review. Am J Prev Med 22(4S):73-107

41. Zaza S, Briss PA, Harris KW (2005) Task force on community. Preventive services. The guide to community preventive services. What works to promote health? Oxford University Press, New York

42. Eden K, Orleans T, Mulrow C et al. (2002) Does counseling by clinicians improve physical activity? A summary for the US Prevention Task Force. Annals Internal Med 137:208-215

43. Hillsdon M, Foster C, Cavill N et al. (2005) The effectiveness of public health interventions for increasing physical activity among adults: a review of reviews. Health Development Agency, London

44. Dishmann AL, Oldenburg B, O’Neal H, Shephard RJ (1998) Worksite physical activity interventions. Am J Prev Med 15:344-361

45. Proper KI, Koning M, van der Beek AJ et al. (2003) The effectiveness of worksite physical activity programs on physical activity, physical fitness, and health. Clin J Sports Med 13:106-117

46. King AC, Rejeski J, Buchner D (1998) Physical activity interventions targeting older adults. Am J Prev Med 15(4):316-333
47. Ashworth NL, Chad KE, Harrison EL et al. (2005) Home versus center based physical activity programs in older adults. The cochrane database of systematic reviews. Issue 1 . Health Development Agency, London

48. Stone E, McKenzie TL, Welk GJ, Booth ML (1998) Effects of physical activity interventions in youth. Review and synthesis. Am J Prev Med 15:298-315

49. Taylor WC, Baranowski T, Young DR (1998) Physical activity interventions in low-income, ethnic minority, and populations with disability. Am J Prev Med 15:334-343

50. Hillsdon M, Foster C, Thorogood M (2005) Interventions for promoting physical activity. The cochrane database of systematic reviews. Issue 1. Art. No.: CD003180. DOI: 10.1002/14651858. CD003180.pub2.

51. Marcus BH, Owen N, Forsyth L et al. (1998) Physical activity interventions using mass media, print media and information technology. Am J Prev Med 15:362-378

52. Marshall AL, Owen N, Bauman AE (2004) Mediated approaches for influencing physical activity: update of the evidence on mass media, print, telephone and website delivery of interventions. J Sci Med Sport 7:74-80

53. Cavill N, Foster C (2004) How to promote healthenhancing physical activity: community interventions. In: Oja P, Borms J (eds) The multidisciplinary series of physical education and sport science. Vol. 6: Perspectives health enhancing physical activity. Meyer \& Meyer, Oxford

54. Marcus BH, Nigg CR, Riebe D, Forsyth LH (2000) Interactive communication strategies. Implications for population-based physical activity promotion. Am J Prev Med 19:121-126

55. Pucher J, Dijkstra L (2003) Promoting safe walking and cycling to improve public health: Lessons from the Netherlands and Germany. Am J Public Health 93:1509-1516

56. Bull FC, Bellew B, Schöppe S, Bauman AE (2004) Developments in national physical activity policy. An international review and recommendations towards better practice. J Sci Med Sport 7(S):93-104

57. Vuori I, Lankenau B, Pratt M (2004) Physical activity policy and program development: The experience in Finland. Public Health Reports 119:331-345 